\title{
CROP DISEASE LEAF IMAGE SEGMENTATION METHOD BASED ON COLOR FEATURES
}

\author{
Lidi Wang*, Tao Yang, Youwen Tian \\ College of Information \& Electrical Engineering, Shenyang Agricultural University \\ * Corresponding author, Address: College of Information \& Electrical Engineering, Shenyang \\ Agricultural University, 120 Dongling Road, Shenyang, 100161, P. R. China, Tel: +86-24- \\ 88487129, Email: wanglidi@gmail.com
}

Abstract: The color feature has been taken an important role in color image segmentation, especially in the fields of automatic detection of crop disease based on leaf image. In this paper an effective method for image segmentation of cucumber leaf images is proposed. First, the color space model is analyzed. Then a kind of color feature is applied to obtain the feature map, which combines RGB model and HSI model. Finally, the morphological method is used to accomplish the image segmentation. This method has been shown effective through experiments.

Keywords: crop disease, color image segmentation, feature extraction, color space

\section{INTRODUCTION}

Image segmentation is an important topic in image processing task. It can effect the progress of the whole recognition which is becoming more and more important in the agricultural automation. For example, in the field of crop disease automatic recognition. According to the crop leaf image, the disease type can be confirmed with the image processing method and expert's knowledge. Howerever, the segmentation's accuracy cannot satisfy the current practical needs. There are primarily four types of segmentation techniques: thresholding, boundary-based, region-based, and hybrid techniques. (Frank Y, 2005) Thresholding is the representative method and depends on the feature map and threshold value. It is based on the assumption that clusters in the histogram correspond to either background or 
objects of interest that can be extracted by separating these histogram clusters. In this paper, combining the RGB model and HSI model, a kind of color feature is applied. Along with the threshold selection and morphological method, an image segmentation method is proposed.

The remainder of this paper is organized as follows. The color space is explained in section II. Section III includes the color feature selection and their characters. Section IV explains the image segmentation method. Finally, the conclusions and discussions of this paper are given in section $\mathrm{V}$.

\section{COLOR SPACE}

Colors are the important feature in color image processing, especially in crop images. Color provides important information for humans to recognize images which can be illuminated under a very wide range of conditions. Commonly used well-known color spaces include (T. Gevers, 1999): (for display and printing processes) RGB, CMY; (for television and video) YIQ, YUV; (standard set of primary colors) XYZ; (uncorrelated features) I1I2I3; (normalized color) rgb, xyz; (perceptual uniform spaces) and (for humans) HSI. Therefore, in this paper, we concentrate on the following standard, color features: intensity I, RGB, hue H and saturation S. Every color space has its advantages and disadvantages.

The HSI color model is cylindrical with the intensity axis coinciding with the achromatic diagonal of the RGB system. Saturation is the radius from the intensity axis and hue is the angle with respect to the red direction. From a perceptual point of view, color can be described in HIS color model by three attributes. The Hue $\mathrm{H}$ is a value which represents the main color of the pixel in the RGB triplet; the saturation S describes the pureness of the color, and I represents the amount of light received by the sensor. It depends on the lighting conditions and on the light source emissivity (Marcos, 1998).

Let R, G and B, obtained by a color camera, represent the 3-D sensor space

$$
C=\int_{\lambda} p(\lambda) f_{c}(\lambda) d \lambda
$$

for $C \in(R, G, B)$, where $p(\lambda)$ is the radiance spectrum and $f_{c}(\lambda)$ are the three color filter transmission functions.

To represent the RGB-sensor space, a cube can be defined on the R, G, and $\mathrm{B}$ axes. White is produced when all three primary colors are at $\mathrm{M}$, where $M$ is the maximum light intensity, say $M=255$. The main diagonal-axis connecting the black and white corners defines the intensity

$$
I(R, G, B)=R+G+B
$$


The transformation from RGB to describe the color impression hue $\mathrm{H}$ is given by

$$
H(R, G, B)=\arctan \left(\frac{\sqrt{3}(G-B)}{(R-G)+(R-B)}\right)
$$

and saturation $\mathrm{S}$ measuring the relative white content of a color as having a particular hue by

$$
S(R, G, B)=1-\frac{\min (R, G, B)}{(R+G+B)}
$$

The saturation is a percentage between 0 and $100 \%$ ( $0 \%$ for achromatic colors as white, greys or black and $100 \%$ for the vivid colors). Hue is measured as an angle between 0 and $360^{\circ}\left(0^{\circ}, 120^{\circ}, 240^{\circ}\right.$ for the basic red R, green $\mathrm{G}$ and blue B). (M. Herbin, 1993)

\section{FEATURE EXTRACTION METHOD}

As analyzed in section 2, varies color features are used in image segmentation. In RGB color space, three features named color mean often be used:

$$
\left\{\begin{array}{l}
\bar{R}=\frac{1}{n} \sum_{i=1}^{n} R_{i} \\
\bar{G}=\frac{1}{n} \sum_{i=1}^{n} G_{i} \\
\bar{B}=\frac{1}{n} \sum_{i=1}^{n} B_{i}
\end{array}\right.
$$

In HSI color space, three parameters $\mathrm{H}, \mathrm{S}$ and I are commonly used to reflect corresponding characters of the image.

Take cucumber disease leaf image as an example. The feature map of original image is computed as shown in figure 1 .

From the figure 1, it is easily to show the pixels in the disease area of the leaf have two distinct characters: one is their $\mathrm{R}$ value is much higher than others, the other is their $\mathrm{H}$ value is much lower than others. So a feature can be defined:

$$
R H=R-H
$$

Using this feature, image segmentation could be accomplished more effectively. The details will be shown in the next section. 


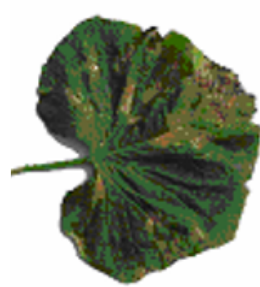

a) the original image

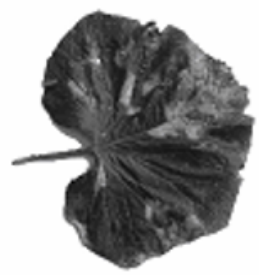

b) $R$ feature map

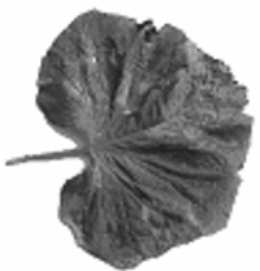

c) G feature map

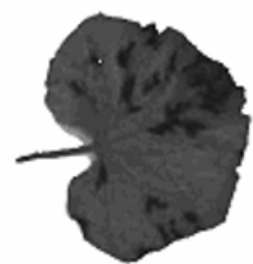

d) H feature map

Fig. 1. Cucumber disease leaf image and its different feature maps

\section{IMAGE SEGMENTATION METHOD}

The details of the color image segmentation method are shown as follows:

Step 1: leaf target extraction. Using the region increasing method to obtain the whole leaf image without the background.

Step 2: computing color feature map. Using the RH feature discussed in the section 3, the feature map can be obtained.

Step 3: threshold selection. Select a suitable threshold value according experience. A binary image can be obtained.

Step 4: morphology processing. Wipe out the little holes and noises by morphological method.

The experiment results are shown as figure 2 .

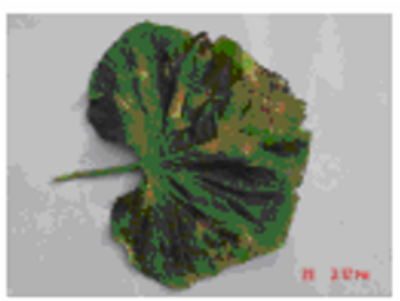

a) the original image

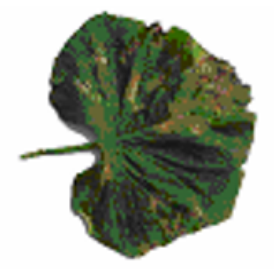

b) leaf target extraction results

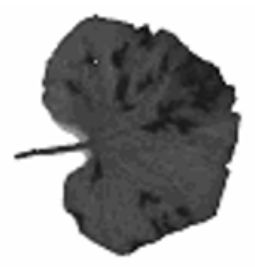

c) RH feature map,

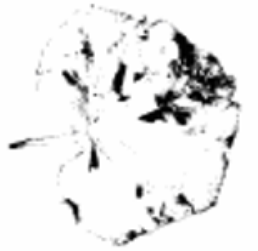

d) thresholding result

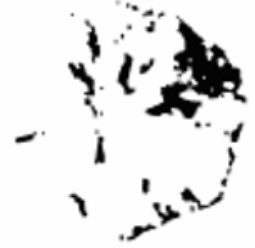

e)morphological processing result.

Fig. 2. Image segmentation results based on the color feature 


\section{CONCLUSION}

Through experiments results it can be concluded that the proposed method can accomplish the image segmentation effectively. Owing to the complex of the disease leaf image's colors, figures and textures, the shortage of this method is the threshold selection method, and this is the hard work in thresholding method of image segmentation. In our experiment, the Otsu algorithm has been used, but cannot obtain the effective result. More effectively automatic threshold value confirming method is the next work to do.

\section{ACKNOWLEDGEMENTS}

This research is supported by Natural Science Foundation of Liaoning Province, China (No. 20052125)

\section{REFERENCES}

Frank Y. Shih, Shouxian Cheng. Automatic Seeded Region Growing for Color Image Segmentation. Image and Vision Computing 2005, 23(10): 877-886.

M. Herbin, F.X. Bon, A., Venot etc. Assessment of Healing Kinetics Through True Color Image Processing. IEEE Transactions on Medical Imaging, 1993, 12(1): 39-43.

Marcos Cordeiro D'Ornellas, Rein Van Den Boomgaard, Jan-mark Geusebroek. Morphological Algorithms for Color Images Based on a Generic-programming Approach. Anais do XI SIBGRAPI, 1998: 1-8.

T. Gevers, A.W.M. Smeulders. Color-based Object Recognition. Pattern Recognition, 1999, 32(11): 453-464. 